\title{
openheart Low incidence of left atrial delayed enhancement with MRI in patients with AF: a single-centre experience
}

\author{
John P Bois, ${ }^{1}$ James Glockner, ${ }^{2}$ Phillip M Young, ${ }^{2}$ Thomas A Foley, ${ }^{1,2}$ \\ Seth Sheldon, ${ }^{1}$ Darrell B Newman, ${ }^{1}$ Grace Lin, ${ }^{1}$ Douglas L Packer, ${ }^{1}$ Peter A Brady ${ }^{1}$
}

To cite: Bois JP, Glockner J, Young PM, et al. Low incidence of left atrial delayed enhancement with MRI in patients with AF: a singlecentre experience. Open Heart 2017;4:e000546. doi:10.1136/openhrt-2016000546

Received 6 October 2016 Revised 13 December 2016 Accepted 18 December 2016

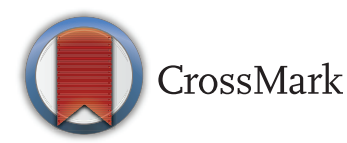

\footnotetext{
${ }^{1}$ Division of Cardiovascular Diseases, Mayo Clinic, Rochester, Minnesota, USA ${ }^{2}$ Department of Radiology, Mayo Clinic, Rochester, Minnesota, USA
}

Correspondence to Dr John P Bois; bois.john@mayo.edu

\section{ABSTRACT}

Background: Atrial fibrillation (AF) is the most common sustained atrial arrhythmia. One potential target for ablation is left atrial (LA) scar (LAS) regions that may be the substrate for re-entry within the atria, thereby sustaining AF. Identification of LAS through LA delayed gadolinium enhancement (LADE) with MRI has been proposed.

Objectives: We sought to evaluate LADE in patients referred for catheter ablation of AF.

Methods: Prospective analysis was conducted of consecutive patients who underwent pulmonary vein antrum isolation (PVAI) ablation for AF at a single institution. Patients underwent LADE with MRI to determine LAS regions before ablation. MRI data were analysed independently in accordance with prespecified institutional protocol by two staff cardiac radiologists to whom patient outcomes were masked, and reports of LADE were documented. Where no initial consensus occurred regarding delayed enhancement (DE), a third staff cardiac radiologist independently reviewed the case and had the deciding vote.

Results: Of the 149 consecutive patients (mean (SD) age, 59 (9) years), AF was persistent in $64(43 \%)$ and paroxysmal in $85(57 \%)$; $45(30 \%)$ had prior ablation. Only five patients $(3 \%)$ had identifiable DE in LA walls (persistent $A F, n=1$; paroxysmal $A F, n=4$ ). LADE was present in two (4\%) of the 45 patients with previous left PVAI. The presence of LADE was not associated with a higher recurrence rate of AF.

Conclusions: In contrast to previous studies, the finding of DE within LA walls was uncommon and, when present, did not correlate with AF type or risk of AF recurrence. It therefore is of unclear clinical significance.

\section{INTRODUCTION}

Atrial fibrillation (AF) is the most common sustained heart rhythm disorder, predicted to affect 12.1 million persons in the USA by 2050. ${ }^{1}$ Current therapeutic options to maintain sinus rhythm in symptomatic AF include pharmacological and non-pharmacological approaches. Recognition that at least some forms of $\mathrm{AF}$ are caused by triggers arising

\section{KEY QUESTIONS}

What is already known about this subject?

- Atrial fibrillation is the most common sustained atrial arrhythmia. A potential target for therapeutic ablation is left atrial (LA) scar areaspotential substrate for re-entry within the atria.

What does this study add?

- LA fibrosis was not detected in the majority of patients with atrial fibrillation undergoing MRI at our institution. Current imaging protocols used by most imaging centres do not provide the ability to detect or quantify LA delayed gadolinium enhancement.

How might this impact on clinical practice?

- If LA delayed gadolinium enhancement could be consistently visualised, quantified and used to prognosticate recurrence of atrial fibrillation, then current practice standards would need to be enhanced.

from the pulmonary veins ${ }^{2}$ has led to more widespread adoption of catheter ablation as an integral approach to treatment of these patients.

Although the success of catheter ablation of paroxysmal AF types is high, success rates of catheter ablation of the more persistent $\mathrm{AF}$ forms is lower-about $50 \%$ at 5 years after a single procedure, with some improvement after recurrent ablative intervention. ${ }^{3}$ Factors affecting the success of catheter ablation of persistent AF forms are complex and poorly understood, and they include frequent and often multiple comorbidities and genetic factors leading to tissue fibrosis and scarring.

Left atrial (LA) remodelling due to myocyte inflammation and LA scar (LAS), particularly in patients with persistent AF, may serve as a substrate for maintenance of micro-re-entry or rotors that drive $\mathrm{AF}$ recurrences. ${ }^{4}$ Therefore, identification of LAS presence and extent in patients who 
present with AF may be useful in risk stratification and selection of best strategy at the time of catheter ablation.

One suggested identification method is to image areas of LAS using cardiac MRI with gadolinium. ${ }^{5}$ Moreover, investigators have proposed that this technique may assist in selection, risk stratification and procedural approach. ${ }^{6}$ Therefore, we sought to evaluate this technique in a prospective cohort of patients undergoing catheter ablation for symptomatic AF at our institution.

\section{METHODS}

\section{Study design}

A prospective cohort of patients undergoing LA ablation for symptomatic AF who underwent MRI preablation and postablation (3 months) with assessment of LA delayed gadolinium enhancement (LADE) were studied. Study protocol was approved by the Mayo Clinic Institutional Review Board and was compliant with the Health Insurance Portability and Accountability Act.

\section{Study population}

A prospective review was conducted of 149 consecutive patients who underwent pulmonary vein antrum isolation (PVAI) ablation between 9 September 2009 and 8 November 2012, at Mayo Clinic in Rochester, Minnesota. Patients with paroxysmal and persistent AF were included and underwent delayed enhancement (DE)-MRI immediately before elective PVAI ablation. Patients with contraindications to DE-MRI (eg, previously implanted cardiac defibrillator or pacemaker, impaired renal function with glomerular filtration rate $<30 \mathrm{~mL} / \mathrm{min} / 1.73 \mathrm{~m}^{2}$, claustrophobia) were excluded. Comprehensive chart review was conducted to gather demographic, medical, cardiac, echocardiographic and laboratory data before PVAI. Patients were considered to have persistent $\mathrm{AF}$ when they had documentation from electrocardiography that was consistent with the American College of Cardiology definition of persistent $\mathrm{AF}$, or $\mathrm{AF}$ sustained beyond 7 days. ${ }^{7}$

\section{Delayed enhancement MRI}

Magnetic resonance angiography of the pulmonary veins was performed to document the number and location of pulmonary veins before ablation. General Electric 1.5-tesla scanner with V.16.0 software and 8-channel phased array receive coil was used to obtain all images. Late gadolinium enhancement (LGE) imaging of the left atrium was also performed to assess for atrial DE. Electrocardiography-gated, two-dimensional inversion recovery spoiled gradient recalled LGE images were acquired in axial and short-axis planes with the following parameters: $5-8 \mathrm{~mm}$ slice thickness with $1 \mathrm{~mm}$ gap; image matrix, 256 $\times 192$; ratio of repetition time to echocardiography time (TR/TE), $3.9 / 1.2 \mathrm{~ms}$; and $32 \mathrm{kHz}$ receiver bandwidth. Breath-held electrocardiographygated, three-dimensional inversion recovery Spoiled gradient recalled acquisition (SGPR) LGE images were acquired in the axial plane with the parameters of 3$5 \mathrm{~mm}$ section thickness; image matrix, 256 $\times 192$; TR/TE, $3.4 / 1.6 \mathrm{~ms}$; and $100 \mathrm{kHz}$ receiver bandwidth. Field of view was adjusted in accordance with patient size (range 32$46 \mathrm{~cm}$ ). Inversion time was selected with an inversion recovery-prepared, multiphasic, electrocardiographygated spoiled gradient recalled sequence performed immediately before LGE image acquisition. Two-dimensional and three-dimensional images were obtained at 10-20 min following injection of $0.2 \mathrm{mM} / \mathrm{kg}$ gadodiamide (Omniscan; GE Healthcare) or gadobenate dimeglumine (MultiHance; Bracco Diagnostics). Nulling of the ventricles was performed during DE imaging. All DE slices from the two-dimensional images were viewed. The three-dimensional volume was reviewed in the twodimensional plane. Each interpreting physician reviewed the studies separately at an independent workstation, with window width and level left to the discretion of the reviewing physician.

Two staff cardiac radiologists (JG and PMY) conducted blinded, independent review of the 149 patients to determine the presence or absence of LADE. A third staff cardiac radiologist (TAF) to whom study details were masked reviewed independently those studies where a consensus was not reached initially regarding the presence of LADE. In these cases, the interpretation by the third radiologist determined whether the study was considered to demonstrate LADE.

\section{Ablation}

The 149 PVAI ablations were performed by two operators. Intracardiac echocardiography was used to examine the pulmonary veins and to guide transseptal catheterisation. In all cases, circumferential ablation to isolate pulmonary vein potentials was performed, followed by mapping and ablation of non-pulmonary vein triggers when found (eg, superior vena cava, vein of Marshal). Linear ablation within the left atrium was generally not routinely performed unless a sustained atrial flutter or recurrent, persistent $\mathrm{AF}$ was observed. Linear ablation of the cavotricuspid isthmus was performed even in the absence of clinically observed typical atrial flutter. In each case, intraprocedural induction and reinduction of $\mathrm{AF}$ through atrial burst pacing with isoproterenol were implemented to detect early recurrences.

\section{Statistical analysis}

Continuous variables are presented as mean (SD) and categorical variables are presented as number and percentage of patients. Univariate logistic regression analysis was performed to analyse the association between clinical variables and the presence or absence of LADE. Pearson $\chi^{2}$ analyses and Fisher exact tests were used as appropriate. Statistical significance was set a priori as $\mathrm{p}<0.05$. All statistical analysis was performed with JMP Pro 9 (SAS Institute). 


\section{RESULTS}

\section{Patient demographic characteristics}

The study included 149 patients (table 1). Mean (SD) age was 59 (9) years; 107 (72\%) were male patients. Of all patients, only five $(3 \%)$ were thought to have evidence of LADE. In contrast, $144(97 \%)$ patients showed no evidence of LADE despite careful prospective review. Mean (SD) age (60 (9) vs $63(10), \mathrm{p}=0.36)$ and race $(97 \%$ vs $100 \%$ white, $\mathrm{p}>0.99)$ were similar across groups. The group with DE had more female patients (100\% vs $26 \%)$.

\section{Medical comorbidities}

In comparison of $\mathrm{AF}$ patients without and with $\mathrm{LADE}$, no statistical significance was found in prevalence of medical comorbidities (table 1), including diabetes mellitus $(\mathrm{p}=0.32)$, hypertension $(\mathrm{p}=0.67)$, hyperlipidaemia $(\mathrm{p}=0.37)$, chronic obstructive pulmonary disease $(\mathrm{p}>0.99)$, prior or current smoking $(\mathrm{p}=0.66)$, sleep apnoea $(\mathrm{p}=0.66)$ and renal function (creatinine level, $\mathrm{p}=0.36)$.

\section{Cardiovascular comorbidities and echocardiographic data}

Sixty-six (44\%) patients were in AF at the time of cardiac MRI acquisition. No statistical significance was found between AF patients without LADE and those with it (table 2) when examining type of $\mathrm{AF}$ (persistent, $\mathrm{p}=0.39$ ), prior AF ablation procedure $(\mathrm{p}=0.63)$, prior myocardial infarction (MI) $\quad(p>0.99)$, coronary artery disease $(p>0.99)$, prior percutaneous coronary intervention or coronary artery bypass graft $(\mathrm{p}>0.99)$, left ventricular ejection fraction $(\mathrm{p}=0.42)$ or history of heart failure $(\mathrm{p}>0.99)$.

\section{Pharmacotherapy}

No statistical difference was found in pharmacotherapy (table 3). Medication use evaluated between patients

Table 1 Baseline characteristics and medical comorbidities of the 149 study patients

\begin{tabular}{|c|c|c|c|}
\hline Characteristics* & $\begin{array}{l}\text { Delayed } \\
\text { enhancement }\end{array}$ & $\begin{array}{l}\text { No delayed } \\
\text { enhancement }\end{array}$ & $\begin{array}{l}p \\
\text { Value }\end{array}$ \\
\hline $\begin{array}{l}\text { Age, mean (SD), } \\
\text { years }\end{array}$ & $63(10)$ & $60(9)$ & 0.36 \\
\hline Female sex & $5(100)$ & 37 (26) & 0.002 \\
\hline White ethnicity & $5(100)$ & $140(9)$ & $>0.99$ \\
\hline Hypertension & $3(60)$ & $68(47)$ & 0.67 \\
\hline Hyperlipidaemia & $1(20)$ & 71 (49) & 0.37 \\
\hline $\begin{array}{l}\text { Smoking (prior } \\
\text { or current) }\end{array}$ & $2(40)$ & $47(32)$ & 0.66 \\
\hline Sleep apnoea & $1(10)$ & 49 (34) & 0.66 \\
\hline COPD & $0(0)$ & $2(1)$ & $>0.99$ \\
\hline $\begin{array}{l}\text { Diabetes } \\
\text { mellitus (type } \\
1 \text { or } 2 \text { ) }\end{array}$ & $1(20)$ & $10(7)$ & 0.32 \\
\hline $\begin{array}{l}\text { Creatinine, } \\
\mathrm{mg} / \mathrm{dL}\end{array}$ & $0.9(0.2)$ & $1.1(1.1)$ & 0.36 \\
\hline
\end{tabular}

Table 2 Cardiovascular comorbidities of the 149 study patients

\begin{tabular}{lllr}
\hline Characteristic* & $\begin{array}{l}\text { Delayed } \\
\text { enhancement }\end{array}$ & $\begin{array}{l}\text { No delayed } \\
\text { enhancement }\end{array}$ & $\begin{array}{l}\text { p } \\
\text { Value }\end{array}$ \\
\hline Persistent AF & $1(20)$ & $66(46)$ & 0.39 \\
Prior ablation & $2(40)$ & $44(31)$ & 0.63 \\
Prior MI & $0(0)$ & $3(2)$ & $>0.99$ \\
CAD & $0(0)$ & $20(13)$ & $>0.99$ \\
Heart failure & $0(0)$ & $13(9)$ & $>0.99$ \\
LVEF & $62(5)$ & $59(7)$ & 0.42 \\
Intervention, & $0(0)$ & $17(11)$ & $>0.99$ \\
stent or bypass & & & \\
\hline
\end{tabular}

${ }^{*}$ Continuous variables expressed as mean (SD); categorical variables are expressed as number and percentage of patients. $A F$, atrial fibrillation; CAD, coronary artery disease; LVEF, left ventricular ejection fraction; MI, myocardial infarction.

Table 3 Pharmacotherapy of the 149 study patients

\begin{tabular}{llcr}
\hline $\begin{array}{l}\text { Medication, } \\
\text { No. (\%) }\end{array}$ & $\begin{array}{l}\text { Delayed } \\
\text { enhancement }\end{array}$ & $\begin{array}{l}\text { No delayed } \\
\text { enhancement }\end{array}$ & $\begin{array}{l}\text { p } \\
\text { Value }\end{array}$ \\
\hline HCTZ & $0(0)$ & $7(5)$ & $>0.99$ \\
ACE inhibitor or & $2(40)$ & $40(28)$ & 0.62 \\
ARB & & $15(10)$ & $>0.99$ \\
Loop diuretics & $0(0)$ & $78(54)$ & 0.66 \\
$\beta$-Blocker & $2(40)$ & $26(18)$ & $>0.99$ \\
CCB & $1(20)$ & $62(43)$ & $>0.99$ \\
Antiarrhythmic & $2(40)$ & $134(93)$ & $>0.99$ \\
Antiplatelet & $5(100)$ & $3(2)$ & $>0.99$ \\
Vasodilator & $0(0)$ & $10(7)$ & $>0.99$ \\
Potassium-sparing & $0(0)$ & & \\
diuretics & & $7(5)$ & 0.24 \\
Digoxin & $1(10)$ & & \\
\hline \multicolumn{4}{l}{ ARB, angiotensin receptor blocker; CCB, calcium channel blocker; } \\
HCTZ, hydrochlorothiazide.
\end{tabular}

without LADE and those with LADE was calcium channel blockers $\quad(p>0.99)$, angiotensin-conversion enzyme inhibitors or angiotensin receptor blockers ( $p=0.62)$, hydrochlorothiazide $(p>0.99)$, loop diuretics ( $p>0.99)$, potassium-sparing diuretics $(p>0.99)$, peripheral vasodilators $(p>0.99)$, digoxin $(p>0.99)$ and antiarrhythmics $(p>0.99)$ or antiplatelets $(p>0.99)$.

\section{Left arterial delayed gadolinium enhancement}

There was agreement between reviewer 1 and reviewer 2 that 121 patients $(81 \%)$ had no evidence of LADE. However, they disagreed regarding the other 28 patients $(19 \%)$ (table 4). These other patients underwent further evaluation by reviewer 3 , who found that five (3\% of the total study population) had LADE. There was no statistical significance in the recurrence rate of atrial fibrillation (defined as atrial fibrillation occurring outside of the postablation 3-month blanking period) between those without DE on preablation MRI (56 $(39 \%)$ recurred) compared with those with DE on preablation MRI (3(60\%) recurred) $(\mathrm{p}=0.34)$. 
Table 4 Presence or absence of LADE

\begin{tabular}{|c|c|c|c|c|}
\hline \multicolumn{3}{|l|}{ Initial review, No. (\%) } & \multicolumn{2}{|c|}{$\begin{array}{l}\text { Review of studies where initial consensus not } \\
\text { reached, No. (\%) }\end{array}$} \\
\hline Delayed enhancement $(n=49)$ & Reviewer 1 & Reviewer 2 & Delayed enhancement $(n=28)$ & Reviewer 3 \\
\hline Yes & 28 (19) & $0(0)$ & Yes & $5(18)$ \\
\hline No & $121(81)$ & $149(100)$ & No & $23(82)$ \\
\hline
\end{tabular}

\section{DISCUSSION}

The present study evaluated the ability of cardiac MRI to detect LADE in the AF population. We used standard imaging techniques and study protocols that reflect current practice at most centres. Although one centre has reported the ability to detect LADE, ${ }^{568}$ the present study shows the difficulty in repeating those observations when using the imaging technology and protocols that are most readily available-and thereby reflect realworld practice. Indeed, in the present investigation, LADE was rarely appreciated even after prior ablation. It remains to be seen whether the dissemination of the imaging techniques that showed detection of LADE at one centre ${ }^{568}$ to multiple sites will enable consistent assessment of LADE. This question is critical given the prior observations that LADE can be readily visualised and can affect prognosis, ${ }^{6} 8$ and ultimately may serve as a target for therapeutic intervention. If this target was the case, then it would be imperative to change the current standard of care practiced at the majority of imaging centres, because this paper shows the inability to detect, let alone quantify, LADE in the AF population when using readily available technology.

Several possible reasons may explain why we observed much less frequent LADE than reported in previous studies. Certainly, patient factors-such as difficulty imaging the thin LA wall (figure 1) and heart rate, as well as differences in imaging protocol-could have a part. For example, Oakes et a $\tilde{\varphi}$ reported finding LADE in all $81 \mathrm{AF}$ patients before they received ablation. A follow-up investigation of 120 patients identified LADE in $\mathrm{AF}$ patients, and quantified the extent of LADE $(<5 \%, 5-20 \%, 20-35 \%$ or $>35 \%){ }^{6}$ Furthermore, that
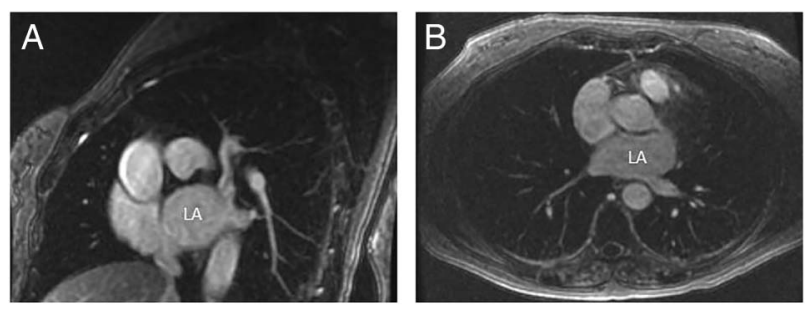

Figure 1 No delayed atrial enhancement on MRI. A 53-year-old woman with a history of paroxysmal atrial fibrillation showed no evidence of left atrial delayed enhancement (LADE) on cardiac MRI before pulmonary vein antrum isolation. Of note, the left atrial wall is thin $(<2 \mathrm{~mm})$, making detection of LADE difficult. (A) Short-axis horizontal view. (B) Horizontal long-axis view. LA, left atrium. study reported that patients with a greater degree of LADE were more likely to have persistent AF, as well as a higher risk of recurrence after ablation. In 2013, in its Delayed Enhancement: MRI Determinant of Successful Catheter Ablation of Atrial Fibrillation Study, ${ }^{8}$ the same centre noted that each increased per cent of fibrosis determined with LADE before ablation was associated with a $6.3 \%$ increase of recurrent symptoms $(\mathrm{p}<0.001)$. For every per cent of residual fibrosis that was detected postprocedure, risk of AF recurrence increased $8.2 \%$ $(\mathrm{p}<0.001)$.

We used standard DE-MRI protocol, which included acquisition in axial and short-axis projections obtained 10-20 min following administration of gadodiamide or gadobenate. The acquisition protocols of studies that showed a greater percentage of patients with LADE have been reported previously. ${ }^{6}$ These protocols included the use of epicardial and endocardial LA border delineation with Seg3D software (National Institutes of Health Center for Integrative Biomedical Computing at University of Utah), LA wall fibrosis quantification with MATLAB software (The MathWorks), three-dimensional MRI visualisation with custom-made image processing software, and a colour lookup table mask to differentiate between enhanced and non-enhanced tissue. These techniques are not used in our evaluation of LADE.

It is plausible that the three-dimensional, navigatorgated LGE sequence, which was used in other investigations, ${ }^{6}$ may maximise spatial resolution and consequently improve visualisation of atrial DE. However, this capability was not available on commercial General Electric systems at the time the present study was performed. Moreover, the imager must be cognisant of the trade-off between greater spatial resolution and signal to noise ratio. Indeed, navigator gating allows greater flexibility in increasing the acquisition time, thereby improving spatial resolution. However, increased imaging time may produce more artefacts because it increases the possibility of patient movement or variants in respiratory patterns.

The longer acquisition time of a navigated sequence also may make it more challenging to perform DE imaging because the inversion times of the tissues can drift significantly over time after gadolinium is injected. It may be technically challenging to perform any DE techniques of the atria because the selection of inversion time may be much more challenging for the thin atrial structure than typical for conventional ventricular DE imaging. How easily the published data for atrial DE 
can be reproduced on scanners provided by different vendors and on different software versions, using different contrast agents, different acquisition parameters and different visualisation tools, remains to be seen.

\section{Study limitations}

The present study found only a small number of patients with LADE through use of imaging parameters that matched the widely used clinical sequences. The small number of positive results may indicate that the study was underpowered to show statistical differences among patients with and without LADE. However, the primary objective of the investigation was to determine whether the detection of LADE could be reproduced readily with standard clinical imaging techniques. Our spatial resolution was $2.5 \mathrm{~mm}$ and therefore may have been limited in ability to resolve smaller structures. Gadobenate dimeglumine persists longer within the blood pool and potentially could have limited atrial wall delineation. Finally, the use of a different MRI vendor (General Electric) with a field strength of 1.5 tesla compared with other sites that may use different vendors, field strengths, imaging parameters and contrast agents may also contribute to the discrepant findings. Nevertheless, the present study is representative of, and therefore applicable to, standard clinical practice.

\section{CONCLUSIONS}

In contrast to previous studies, we did not observe a high incidence of LADE while using standard clinical scanners and typical pulse sequence parameters. Indeed, even with addition of atrial segmentation and enhancement analysis software, the detection of LADE when there is minimal, if any, visual evidence of atrial DE would be challenging. This challenge is compounded further in the AF population, where motion artefacts in electrocardiography-gated MRI further limit DE detection capability, particularly in such a thin structure as the left atrium. Finally, even when it was found in the present study, LADE did not correlate with type of $\mathrm{AF}$ or risk of $\mathrm{AF}$ recurrence and therefore was of unclear clinical significance. Ultimately, this study demonstrated that current imaging protocols used by the majority of imaging centres do not demonstrate the ability to detect or quantify LADE. This finding is critical because if LADE can be consistently visualised, quantified and used to prognosticate recurrence of $\mathrm{AF}$, then current practice standards will need to be enhanced and further resources employed.
Contributors JPB, SS and DBN were responsible for data gathering. JG, PMY and TAF are responsible for LADE detection. PAB and JPB were responsible for study design. DLP and GL were responsible for review of the study and input on revisions. All authors were responsible for manuscript development.

Funding This research received no specific grant from any funding agency in the public, commercial or not-for-profit sectors.

\section{Competing interests None declared.}

Ethics approval This study was approved by Mayo Clinic Institutional Review Board and was compliant with the Health Insurance Portability and Accountability Act.

Provenance and peer review Not commissioned; externally peer reviewed.

Data sharing statement No additional data are available.

Open Access This is an Open Access article distributed in accordance with the Creative Commons Attribution Non Commercial (CC BY-NC 4.0) license, which permits others to distribute, remix, adapt, build upon this work noncommercially, and license their derivative works on different terms, provided the original work is properly cited and the use is non-commercial. See: http:// creativecommons.org/licenses/by-nc/4.0/

\section{REFERENCES}

1. Miyasaka Y, Barnes ME, Gersh BJ, et al. Secular trends in incidence of atrial fibrillation in Olmsted County, Minnesota, 1980 to 2000, and implications on the projections for future prevalence. Circulation 2006;114:119-25.

2. Haïssaguerre $M$, Jaïs $P$, Shah DC, et al. Spontaneous initiation of atrial fibrillation by ectopic beats originating in the pulmonary veins. N Engl J Med 1998;339:659-66.

3. Ganesan AN, Shipp NJ, Brooks AG, et al. Long-term outcomes of catheter ablation of atrial fibrillation: a systematic review and meta-analysis. J Am Heart Assoc 2013;2:e004549.

4. Narayan SM, Krummen DE, Shivkumar K, et al. Treatment of atrial fibrillation by the ablation of localized sources: CONFIRM (Conventional Ablation for Atrial Fibrillation With or Without Focal Impulse and Rotor Modulation) trial. J Am Coll Cardiol 2012;60:628-36.

5. Oakes RS, Badger TJ, Kholmovski EG, et al. Detection and quantification of left atrial structural remodeling with delayed-enhancement magnetic resonance imaging in patients with atrial fibrillation. Circulation 2009;119:1758-67.

6. Akoum N, Daccarett M, McGann C, et al. Atrial fibrosis helps select the appropriate patient and strategy in catheter ablation of atrial fibrillation: a DE-MRI guided approach. J Cardiovasc Electrophysiol 2011;22:16-22.

7. European Heart Rhythm Association; Heart Rhythm SocietyFuster V, Rydén LE, Cannom DS, et al. American College of Cardiology; American Heart Association Task Force on Practice Guidelines; European Society of Cardiology Committee for Practice Guidelines; Writing Committee to Revise the 2001 Guidelines for the Management of Patients With Atrial Fibrillation. ACC/AHA/ESC 2006 guidelines for the management of patients with atrial fibrillation: executive summary: a report of the American College of Cardiology/ American Heart Association Task Force on Practice Guidelines and the European Society of Cardiology Committee for Practice Guidelines (Writing Committee to Revise the 2001 Guidelines for the Management of Patients With Atrial Fibrillation). J Am Coll Cardiol 2006;48:854-906. Erratum in: J Am Coll Cardiol. 2007;50:562.

8. Marrouche NF, Wilber D, Hindricks G, et al. Association of atrial tissue fibrosis identified by delayed enhancement MRI and atrial fibrillation catheter ablation: the DECAAF study. JAMA 2014;311:498-506. Erratum in: JAMA. 2014;312:1805. 